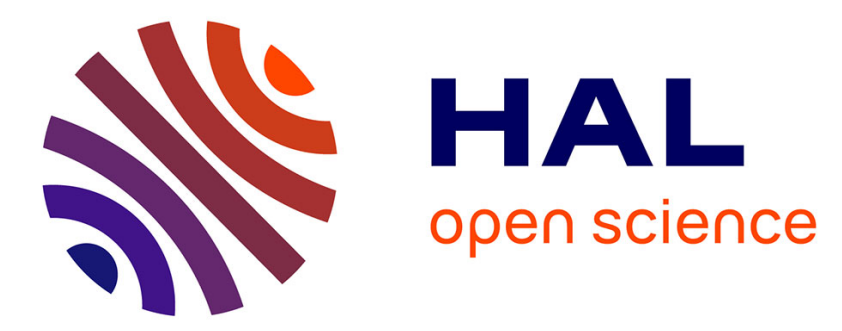

\title{
Impedance flow cytometry allows the early prediction of embryo yields in wheat (Triticum aestivum L.) microspore cultures
}

Julie Canonge, Murielle Philippot, Catherine Leblanc, Philippe Potin, Manuelle Bodin

\section{To cite this version:}

Julie Canonge, Murielle Philippot, Catherine Leblanc, Philippe Potin, Manuelle Bodin. Impedance flow cytometry allows the early prediction of embryo yields in wheat (Triticum aestivum L.) microspore cultures. Plant Science, 2020, 300, pp.110586. 10.1016/j.plantsci.2020.110586 . hal-03020277

\section{HAL Id: hal-03020277 \\ https://hal.sorbonne-universite.fr/hal-03020277}

Submitted on 23 Nov 2020

HAL is a multi-disciplinary open access archive for the deposit and dissemination of scientific research documents, whether they are published or not. The documents may come from teaching and research institutions in France or abroad, or from public or private research centers.
L'archive ouverte pluridisciplinaire HAL, est destinée au dépôt et à la diffusion de documents scientifiques de niveau recherche, publiés ou non, émanant des établissements d'enseignement et de recherche français ou étrangers, des laboratoires publics ou privés. 


\title{
Impedance flow cytometry allows the early prediction of embryo yields in wheat (Triticum
} aestivum L.) microspore cultures.

Julie Canonge, ${ }^{\mathrm{a}}$ Murielle Philippot, ${ }^{\mathrm{a}^{*}}$ Catherine Leblanc, ${ }^{\mathrm{b}}$ Philippe Potin, ${ }^{\mathrm{b}}$ Manuelle Bodin ${ }^{\mathrm{a}}$

${ }^{a}$ Vegenov, Pen ar Prat, 29250 Saint-Pol-de-Léon, France

${ }^{\mathrm{b}}$ CNRS, Sorbonne Université Sciences, UMR 8227, Integrative Biology of Marine Models, Station Biologique de Roscoff, CS 90074, 29688 Roscoff, France

* Corresponding author:

E-mail address: philippot@vegenov.com

\begin{abstract}
Haplomethods are key biotechnological tools that make it possible to rapidly produce perfectly homozygous lines, speeding up plant breeding programs. Under specific stress conditions, microspores are reprogrammed toward sporophytic pathways, leading to embryo formation. Various endogenous and exogenous factors affect embryo yield in androgenesis, so the improvement of androgenesis efficiency requires the development of early, reliable and robust reactivity markers. During the last decade, numerous cytological, cellular and biochemical approaches were carried out to finely characterize microspore development and fate during androgenesis. However, the different available markers are often species-dependent, and their development and application are time-consuming and cumbersome. In this study, we show the suitable use of impedance flow cytometry (IFC) to develop new robust, reliable and strong markers of androgenesis reactivity in wheat, leading to: (i) routine monitoring of the viability of heterogeneous cell cultures; (ii) quick and simple evaluation of stress treatment efficiency; and (iii) early prediction of embryo yields from microspore suspensions. IFC can therefore provide the fine characterization of all of the microspore developmental pathways that occur in a cell suspension, for embryogenic microspores as well as pollen-like microspores. IFC technology has become a very useful tool to track and characterize wheat microspores in androgenesis, but can also be adapted to other species and other in vitro cell culture systems.
\end{abstract}

\section{Keywords}

Androgenesis, microscopy, microfluidics, viability, developmental stage, reactivity marker 


\section{Introduction}

Plant breeding programs are currently integrating haplomethods in order to quickly obtain homozygous plants and to thus reduce the time required for commercial registration of new cultivars [1]. The production of doubled haploids (DH) has therefore become a key tool for the improvement of the breeding process. Since the first report of microspore-derived embryos from Datura anther culture in 1964 [2], androgenesis has been applied to a wide range of plant species [3]. This technique is based on a microspore switch from the gametophytic pathway, resulting in mature pollen formation, towards the sporophytic pathway that leads to embryo production. This switch results from the application of a suitable stress on microspores at a precise developmental stage. Thus, all microspores, from the mid-uninucleate to the early binucleate stage, depending on the species, are potentially capable of forming embryos [4,5], but only a minority of them actually acquire this embryogenic competence. In Brassica, a heat shock from 30 to $35^{\circ} \mathrm{C}$ is usually applied [6,7], while a cold treatment or an osmotic shock is used for many cereals. In the wheat androgenesis process, the stressed microspores are then plated and require ovary co-culture to survive and develop in in vitro culture [8,9]. Throughout the process, androgenesis efficiency is influenced by many factors such as the quality and physiology of donor plants [10], genotype [11], the developmental stage of microspores [12], stress-inducing pretreatment [13] and culture conditions [14]. The achievement of this technique lies in the success of embryogenesis induction as well as in the regeneration of doubled haploid plants. Consequently, DH protocol development and optimization can be difficult, time-consuming and expensive.

In addition to being an essential tool for breeders, androgenesis, in particular, is an interesting way to study embryogenesis without the interactions of seed tissues, allowing the exploration of the underlying plant development process [15]. Isolated microspore cultures allow an easy monitoring of microspores and their development, becoming a model to investigate embryogenesis, particularly in the early stages, in numerous cellular and molecular studies [1619]. During the androgenesis process, many cellular and structural changes are visible in wheat. First, an enlargement of certain cells is visible and cytoplasmic strands connecting both perinuclear and subcortical cytoplasms fragment the vacuole. The nucleus displays a central position. These so-called star-like microspores are a transient and characteristic stage that attest to the embryogenic potential of wheat microspores [5,20-22] and can be found in other plant species such as rice [23], rapeseed [24] and tobacco [4,25]. Despite this, it has been reported that star-like microspores do not always produce androgenic structures in barley microspore suspensions [21,26,27]. Embryogenic microspores are also subjected to changes in the 
cytokinesis pattern, with their phragmoplast rearrangement allowing a first symmetrical division. This specific division is the first indicator of the onset of sporophytic development, in contrast with the first asymmetric division during pollen development $[5,7,18,22,28]$. However, various studies have reported that both symmetric and asymmetric first division could occur in embryogenic microspores, leading to embryo formation [27,29-31], including in wheat anther cultures [5,32]. In that case, wheat embryogenic microspore nuclei actively divide and form multinucleate structures, becoming an androgenic marker easily observable in microscopy. However, depending on the species and genotypes, multinucleate cells can be relatively poorly represented within cultures and therefore difficult to count. Androgenic microspores can also be characterized by the presence of a preprophasic band, absent in pollen, marking the position of the future cell wall [18,33]. In Brassica napus, studies highlight that the intine thickness increase, the specific changes in the cell wall composition and the formation of the osmoprotective subintinal layer occur only in embryogenic microspores at different developmental stages [17,28,34-36].These numerous morphological, structural and biochemical alterations can be used as markers to finely study the embryogenic development of microspores and to improve embryo yields. To track and elucidate intracellular changes that occur during microspore embryogenesis, these approaches require various microscopic approaches and are sometimes coupled with sample fixation or monolayer microspore culture $[5,26,37]$. However, these methods remain cumbersome, time-consuming and complex to set up.

Impedance flow cytometry (IFC) is based on the analysis of the dielectric properties of cells by application of an alternating current. This technique uses microfluidic chips containing electrodes and a microchannel of various sizes, coupled to a Coulter system. The electrode geometry, the microchannel, the buffer conductivity and the application of alternating current at adjustable frequencies first determine the system impedance. When crossing the microchannel, cell suspension modifies the system impedance, reflecting the cells' intrinsic dielectric properties. Depending on the frequency used, ranging from 0.5 to $30 \mathrm{MHz}$, IFC provides different information levels, from cell size to intracellular properties, and cell viability [38]. Many studies have been carried out using IFC on microorganisms to determine cell viability and the size of yeast in bacteria suspensions [39-41], and to study tumor cell differentiation, apoptosis and necrosis [39,42-44]. IFC was recently introduced into plant biology research and is currently used for pollen viability analysis by breeders. This technology has also proven itself to be efficient to detect pollen developmental stages, from tetrad to mature trinucleate pollen in tobacco [45]. Xu et al. [46] identified the QTL qPV11 implicated in the 
heat tolerance of tomato pollen using IFC to phenotype the pollen viability. Moreover, IFC also detected abnormal pollen development in the case of pollen sterility of potato [47]. Likewise, this technology assesses pollen quality by determining germination capacity and pollen viability for many species such as tomato, cucumber and sweet pepper. Heidmann et al. [45] recently demonstrated that the use of IFC as a predictive tool for tomato pollen germination is possible. This technology, non-invasive and label-free, thereby covers vast application fields in cell biology.

110 Using IFC analyses, we investigated the possibility to characterize the different developmental pathways occurring during wheat microspore in vitro culture. The goal was to develop a new marker to allow the early prediction of androgenic embryo yield in a quick, reliable and precise manner.

\section{Material and Methods.}

\subsection{Plant material and growing conditions}

Seeds of the spring wheat (Triticum aestivum L.) genotype Pavon were germinated and cultivated in a growth chamber for two weeks $\left(17^{\circ} \mathrm{C}\right.$ night, $19^{\circ} \mathrm{C}$ day with a 16 -h light period and $70 \%$ relative humidity).

Seedlings were then transferred to four-liter pots (TREF, EGO $N^{\circ} 120$ ), fertilized (Horti-Cote $®$ Plus CRF 15-6-11) and grown in a growth chamber under controlled conditions until tillering $\left(12^{\circ} \mathrm{C}\right.$ night, $18^{\circ} \mathrm{C}$ day, with a 16 -h light period and $60 \%$ relative humidity).

\subsection{Tiller harvest, pretreatment and microspore culture}

Tillers were harvested approximately eight weeks after the seedlings, when the majority of anthers contained mid- to late-uninucleate stage microspores. The microspore developmental stages were checked with DAPI staining [48].

Tillers were placed in jars filled with sterile water and cold pre-treated at $4^{\circ} \mathrm{C}$ for 21 days in the dark, according to the method used by De Buyser et al. [49].

130 After pretreatment, spikes were sterilized and microspores were isolated and cultured according to Zheng et al. (modified) [50]. Ovary co-culture was carried out with two ovaries/mL of microspore suspension [49]. Cultures were incubated at $28^{\circ} \mathrm{C}$ in the dark for 30 days. The embryo yield was established at 30 days by counting the number of embryos per 10,000 microspores.

\subsection{Sample preparation for IFC measurement}


IFC analyses were done on samples collected 21 days before plating (D-21), just before plating (D0), and 1, 5 and 7 days after plating (D1, D5 and D7).

At D-21, microspore suspension was prepared from four freshly collected spikes to produce the baseline IFC data on microspores before androgenesis. Other samples were produced by collecting microspores from suspension cultures.

Samples were prepared immediately before IFC analysis: $1 \mathrm{~mL}$ of microspore suspension was filtered with a $70-\mu \mathrm{m}$ filter (pluriSelect), and $2 \mathrm{~mL}$ of IFC buffer were added to each sample (AF4, Amphasys). An aliquot of $200 \mu \mathrm{L}$ of each sample was used for microscopic observations. To obtain non-viable controls that would allow a clear discrimination between viable and nonviable cells in IFC analysis, heat-treated samples were prepared and analyzed for each measurement. One $\mathrm{mL}$ of microspore suspension was heated at $90^{\circ} \mathrm{C}$ for $7 \mathrm{~min}$ with a thermoshaker (Grant-Bio). After cooling down to room temperature, the heat-treated samples were processed, as previously explained.

\subsection{IFC analyses}

Microspore suspensions were analyzed using the impedance flow cytometer device AmphaZ32 (Amphasys, Switzerland) at 0.5 and $12 \mathrm{MHz}$. Parameter settings were: trigger level: $0.1 \mathrm{~V}$; modulation: 5; amplifier: 5; demodulation: 0; and pump: $80 \mathrm{rpm}$. A 120- $\mu \mathrm{m}$ channel chip was used. Data were analyzed with Amphasoft, version 2.1.5.0.

Integrated values of microspore impedance are represented in a graph with the phase angle in the $\mathrm{x}$-axis and the amplitude in the $\mathrm{y}$-axis. Various gatings such as vertical and polygons can be drawn to categorize each cell set, depending on these two parameters.

In the present study, we defined and analyzed different types of variables calculated by the software. Cell viability, expressed in percentage of viable cells, was calculated each day of analysis from seven independent microspore cultures. Polygons, referred to as P1, P2 and P3, were drawn manually using the gating tool. Each dot plot inside a polygon can be characterized by its phase angle median and amplitude median, automatically calculated by Amphasoft. These medians represent the central value of the dot plot in the polygon. In this study, the variables, referred to as "phase angle median" and "amplitude median", correspond to means of medians from a minimum of five microspore suspensions with three technical replicates. The percentage of viable microspores out of the total number of cells was calculated for each polygon. 
Three different methods were used in visible light microscopy for microspore observations. Microspores were observed: (1) without staining; (2) with fluorescein diacetate (FDA) staining; or (3) with 4,6-diamidino-2-phenylindole, dihydrochloride (DAPI) staining. An inverted microscope (Olympus CKX41) and a microscope equipped for fluorescence illumination (Olympus BH-2) were used. A solution of $100 \mu \mathrm{L}$ of DAPI $\left(4 \mathrm{mg} \cdot \mathrm{mL}^{-1}\right)$ or FDA $\left(41.10^{-3} \mathrm{mg} \cdot \mathrm{mL}^{-}\right.$

${ }^{1}$ ) [51] were added to $200 \mu \mathrm{L}$ of microspore samples prepared as explained above, and at least 200 microspores per sample were counted.

DAPI-stained microspores were classified as uninucleate, binucleate, trinucleate and multinucleate, and the percentage of each category was calculated. Cell viability was estimated using FDA staining, as a percentage of fluorescent microspores out of the total number of cells counted. The percentage of star-like microspores within the population was determined using light microscopy at D0 only.

\subsection{Statistical analyses}

185 Seven biological replicates of microspore suspension culture were performed in this study and technical triplicates were done for each IFC measurement.

For statistical analyses, we used R software, version 3.5.0, with the Rcmdr interface and the missMDA package in $\mathrm{R}$ [52] to replace missing entries with plausible values.

\section{Results}

\subsection{IFC measures cell viability in microspore suspensions.}

Impedance flow cytometry (IFC) allows us to characterize cells according to their dielectric properties when they are subjected to alternating currents at different frequencies. In the first step, to discriminate viable and non-viable cells, microspores in culture medium were sampled, diluted in analysis buffer and analyzed by IFC at $12 \mathrm{MHz}$. These microspore populations were collected at different times, before and during cell culture, from D-21 to D7.

Aliquots of each sample were heat-treated to inactivate microspores and analyzed as negative controls. In the heat-treated samples, the non-viable microspore population was characterized by a homogenous IFC signal with a low phase angle and amplitude (Figure 1A). In the untreated samples, two discrete populations coexisted: one with a low phase angle and amplitude, and the other with a high phase angle and amplitude (Figure 1B). We therefore referred to the population with a low phase angle as the dead microspores and the population with a high phase angle as the viable ones. 
In the second part of the experiment, a series of cell population mixtures was prepared by mixing a fresh and heat-treated microspore suspension to obtain a range from $0 \%$ to $90 \%$ of viable microspores. The 15 resulting samples were analyzed with two types of cell viability measurements: IFC at $12 \mathrm{MHz}$ and FDA staining. A strong correlation between these two sets of measurements was found with $\mathrm{r}^{2}=0.99$ (Figure 1C).

These results demonstrate that IFC technology allows cell viability measurements of microspore suspensions in culture medium, from tiller harvesting until day 7 of in vitro culture.

\subsection{IFC detects different microspore developmental stages during pollen formation.}

IFC analyses were carried out on the gametophytic development of wheat pollen in order to identify their impedance characteristics. We collected microspores at different developmental stages, from mid-to-late uninucleate to mature trinucleate, from different sized tillers. Samples were prepared in the same conditions as previously described and the developmental stages were checked by microscopy before IFC measurement.

Early to late binucleate microspores (Figure 2 EB, LB) showed a significant increase in phase angle compared to uninucleate microspores (Figure 2 ML/D-21). Conversely, the final stages of gametophytic development displayed a decrease in phase angle (Figure 2 ET, LT). We also noticed an increase in the amplitude signal during pollen formation (Figure 2 ET, LT), which can be explained by the increase in microspore size, as observed in microscopy (Figure $\mathbf{3} \mathbf{A}$, B, C). The phase angle and amplitude variations appeared to reflect cellular and cytoplasmic changes occurring within microspores, such as meiosis events, cytoplasmic activity and cell size increase. IFC therefore made it possible to monitor microspore development into mature pollen.

\subsection{IFC detects different microspore development stages during androgenesis.}

Microspore suspensions were analyzed by IFC throughout the androgenesis process, highlighting significant impedance variations (Figure 2 D-21 to D7). To characterize these variations, three polygons were determined manually and in a non-overlapping manner with Amphasoft interface at three precise stages of the androgenesis process. These stages were: spike collecting (D-21), microspore isolation (D0), and after five days of in vitro culture (D5). For each polygon, phase angle and amplitude medians of all microspores present inside were averaged and used to characterize the corresponding microspore population.

The first identified polygon was called P1. It defined the viable microspore population present at the beginning of the androgenesis process at D-21. Phase angle and amplitude medians of 
viable cells present in this polygon P1 were $240 \pm 8$ and $0.6 \pm 0.1$, respectively. After stress application for androgenesis induction (i.e., pretreatment), viable microspores exhibited impedance variations from D0 to D7. First, some microspores showed an increase in phase angle at D0. This population was specified by a P2 polygon with phase angle and amplitude medians of $315 \pm 4$ and $0.7 \pm 0.1$, respectively. Secondly, throughout the culture, some of the viable microspores displayed a continuous increase in phase angle but also in amplitude. This cell population was characterized by a $\mathrm{P} 3$ polygon and by phase angle and amplitude medians of $354 \pm 1$ and $2.4 \pm 0.4$, respectively.

The phase angle and amplitude medians presented above were calculated and determined the day at which the polygon was defined: at D-21, D0 and at D1 for polygons P1, P2 and P3, respectively. Statistical analysis revealed that the phase angle medians are significantly different for the three polygons, while only the P3 amplitude median is significantly different from the others (Figure $4 \mathbf{A}$ and B). We thus assumed that these impedance phase angle and amplitude variations reflect intracellular changes occurring within microspores during androgenesis, and could therefore be associated with biological phenomena.

\subsection{Impedance variations express morphological and intracellular changes occurring within} the microspores during androgenesis.

\subsubsection{IFC shows a decrease in microspore viability during the androgenesis process.}

Microspore viability is an important parameter to monitor during the androgenesis process in order to control the quality of cell suspensions as well as the impact of the process factors. At the spike collecting stage (D-21), cell viability was $81.90 \%$ on average (Figure 5). Cell viability decreased by more than half during stress treatment under these experimental conditions (between D-21 and D0). After one day of in vitro culture (D1), no significant variation in cell viability was observed. In contrast, it significantly decreased after D1, reaching $10.4 \%$ at D7.

\subsubsection{Microscopic characterization of intracellular changes occurring in microspores during} androgenesis.

In addition to IFC measurements, microscopic studies were carried out on the wheat microspore developmental pathways during the androgenesis process (Figure 3).

At D-21, the microspores observed with DAPI staining were mainly at the mid- to lateuninucleate stage, the preferential developmental stage in wheat for androgenesis induction.

Microscopic observations after stress treatment at D0 showed morphological changes within some microspores, and different types of development were visible. One part of the microspores 
did not seem to develop during pretreatment: these cells had a single nucleus and no vacuolization was visible (Figure $3 \mathbf{A}$ ). Another part of the microspores showed one or two nuclei in a surrounding position due to the presence of a large vacuole (Figure 3 B). A final microspore subpopulation exhibited a star-like morphology: significant vacuolization with cytoplasmic strands crossing through the vacuoles and connecting perinuclear and subcortical cytoplasms (Figure 3 D). Their nucleus was in a central position. During the first seven days of culture, two new types of microspores appeared: trinucleate microspores with three more or less elongated nuclei (Figure $3 \mathbf{C}$ ), and multinucleate microspores characterized by a nuclei number equal to or greater than four (Figure $3 \mathbf{E}$ ). Multinucleate microspores exhibited a gradual disappearance of vacuoles and the potential appearance of small vesicles within this cell type. Plasmolyzed cell (Figure 3 F) frequency increased throughout the culture, and less and less cells had intact nuclei visible in microscopy (data not shown).

Microspores were categorized according to their nuclei number and cytological characteristics, and the distribution of cells in each category was calculated and expressed in percentage in Figure 6 A. From D-21 to D0, the proportion of uninucleate microspores dropped to approximately $78 \%$, while the proportion of binucleate cells was multiplied by approximately 10 compared to D-21. Uninucleate and binucleate microspore proportions then remained stable during the first seven days of in vitro culture. Trinucleate and multinucleate microspore proportions never exceeded $1.5 \%$. These forms of cell development remained rare events. Starlike microspores, a transient form of cell development, represented $41.5 \%$ of the population at D0. They were only counted at D0, even if they were still visible during the first days of culture.

\subsubsection{Early biological changes occurring within microspores explain impedance variations} during androgenesis.

Data from IFC analyses were compared to microscopic observations to improve the understanding of impedance variations observed in microspores during the androgenesis process (Figure $6 \mathbf{A}$ ).

300 IFC measurements performed at D-21 showed that P1 polygons contained a large majority of the viable microspores, about $80 \%$ of the $81.90 \%$ viable cells. As observed using microscopy, cells were mostly uninucleate microspores. From D0 and throughout the culture, the percentage of viable microspores in the P1 polygon then decreased to reach $2 \%$, whereas microscopic examinations revealed that the proportion of uninucleate cells remained stable during in vitro culture. 
The majority of viable microspores at D0, about 33\%, were contained in the P2 polygon. This proportion gradually decreased during microspore culture. D0 is a precise and important step in androgenesis and marks the transition between stress treatment, allowing the microspore switch towards the sporophytic pathway and in vitro culture required for embryogenic structure development. Comparison of the percentage of viable microspores in the P2 polygon and starlike microspore proportion at D0 showed a significant correlation $\left(\mathrm{r}^{2}=0.88\right)$ between these two variables (Figure 6 B). The slope value of the correlation line is 0.82 and can be explained by the fact that no distinction is made between viable and non-viable microspores by light microscopy, unlike IFC analysis.

These results show that IFC can very precisely discriminate the different developing cell populations during the early stages of androgenesis. Moreover, IFC measurement at D0 is a potentially reliable predictor for microspore suspension reactivity.

\subsubsection{The percentage of viable cells in the P3 polygon measured by IFC provides an early} prediction of embryo yield.

To investigate microspore culture characterization by IFC, we studied the evolution of cells contained in polygon P3 from D1 to D7.

The highest proportion of viable microspores in the P3 polygon was reached at D1 (10\%) but this proportion then dropped during culture as mortality increased (Figure 6 A). However, the phase angle and amplitude of the microspore population in the P3 polygon increased throughout microspore culture (Figure 2 D1 to D7).

Contrary to expectations, no correlation was found between the percentage of viable microspores in the $\mathrm{P} 3$ polygon and the proportion of multinucleate microspores (data not shown). By comparing the IFC data of wheat gametophytic and sporophytic developments (Figure 2), we showed that no viable microspore was present in the P3 polygon during pollen formation, regardless of the gametophytic developmental stage. These results allowed us to conclude that during the androgenesis process, the cells present in the P3 polygon did not correspond to microspores developing into pollen. As previously stated, multinucleate microspores remain a very rare event to be observed by microscopy. In contrast, with IFC analysis, an average of 8,283 cells per sample was counted and an average of about 293 microspores were present in the P3 polygon at D7. The absence of correlation could therefore be explained by technical constraints based on the low number of cells counted by microscopy, insufficient to characterize the real composition of microspore populations in culture. It was 
therefore crucial to implement another approach to characterize the microspore population in the $\mathrm{P} 3$ polygon.

We decided to set up a novel statistical approach to try to connect the microspores in the P3 polygon with the reactivity level of a microspore suspension. For this advanced approach, we used classic mathematical model methods: first, a generalized linear model to predict embryo production and, second, a linear model for embryo yield prediction. We decided to fix a minimal value for cell culture reactivity for the Pavon genotype at 0.3 embryos per 10,000 microspores. Only four of the seven biological replicates showed an acceptable level of reactivity and were used to further construct the mathematical procedure.

First, we investigated if embryo production, or lack of embryo production, could be predicted by IFC data. Statistical analysis (PCA) showed that the percentage of viable microspores in the P3 polygon at D7 was the best predictive variable for embryo production. Thus, if the percentage of viable microspores in the P3 polygon at D7 exceeds the threshold of $3.1 \%$, the microspore suspension will produce embryos (data not shown).

Secondly, we established a mathematical model based on this same variable to predict embryo yield of a microspore suspension. In this linear model, if the percentage of viable microspores in the $\mathrm{P} 3$ polygon at $\mathrm{D} 7$ exceeds $3.1 \%$, as previously stated, then the embryo production number by a microspore suspension can be predicted by the equation: $y=6.539 x-20.827$, with $y$ corresponding to the number of embryos per 10,000 microspores and $x$ corresponding to the percentage of viable microspores in the P3 polygon at D7 (Figure 7). When applied to our four biological replicates, this mathematical model presented a high significance level $\left(r^{2}=0.95\right.$ and $\mathrm{p}$-value $=0.02)$.

\section{Discussion}

The first research work using IFC analyses on plant cells was published in 2016 by Heidmann et al. [45]. It reported viability measurement of pollen from different plant species at different stages of development, from tetrad to mature pollen. In our study, we demonstrated that microspore suspension viability can also be measured by IFC during the early stages of the androgenesis process. In addition, the sample preparation method described here for the first time allows microspore analysis in their own in vitro culture medium. This novel methodology preserves microspore viability during the sample preparation for IFC analysis and therefore eliminates the potential biases of damaged cells.

We easily monitored microspore viability evolution using IFC, from tiller harvesting to the first seven days of culture. The decrease in cell viability observed in our study is in agreement with 
other studies carried out on several isolated microspore culture systems [53-55]. The first viability decline (from D-21 to D0) may be associated with stress treatment effects. The inductive stress applied to microspores induces several protection and stress-response processes such as heat shock protein expression, proteolytic machinery activation and autophagy $[16,56]$. Studies showed that in barley microspore cultures, autophagy is induced after pretreatment and could be responsible for stress-induced cell death within these microspores [54,55,57]. However, this stress is fundamental for the microspore switch towards the sporophytic pathway [4]. It is therefore necessary to adapt the stress conditions to allow a maximum of microspore reorientation, while preserving their integrity and viability. A second viability drop is observed during microspore culture, corresponding to the degeneration and death of non-responsive and non-embryogenic microspores [27]. Thanks to viability measurement by IFC, it is now possible to multiply stress condition tests and easily evaluate their impact on cell culture viability. IFC is therefore an advantageous tool for routinely monitoring cell viability.

During in vitro culture, microspores arrest their development, continue on a gametophytic pathway leading to pollen formation, or switch to a sporophytic program. Among the responsive microspores, some of them stop their development after only several divisions, whereas the others develop into androgenic structures [27]. After pretreatment, the coexistence of various cell fates and types within the same microspore suspension makes it widely heterogeneous. The separation of these different structures during the early androgenic stages remains difficult at this time [16,58]. IFC allows quick and simple cell population description on heterogeneous populations of isolated microspores in culture without cell sorting.

In this paper, we highlighted amplitude and phase angle increases in microspore suspensions during the androgenesis process and connected these impedance variations to well-known cellular mechanisms described in microspore embryogenesis. Responsive microspores undergo considerable morphological changes as well as a significant cytological reorganization [18]. The embryogenic development of microspores can actually be divided into three phases: acquisition of embryogenic potential, multinucleate structure development and embryo 400 formation [21]. Our results can be linked to the two first phases. We show that the first intracellular changes occurring in androgenesis are reflected by the significant increase in the impedance phase angle of a proportion of viable microspores. At this stage, responsive microspores are subjected to profound modifications of their genetic machinery with the repression of genes related to a gametophytic program and, conversely, the initial overexpression of stress-resistant genes and genes associated with embryogenic development [22,35,59]. These microspores display a weak metabolic activity [28], as reflected by the 
presence of cytoplasmic regions devoid of organelles and the decrease in ribosome density $[21,60]$. We demonstrate in this paper that the acquisition of embryogenic potential, represented by star-like microspores in wheat, is highly correlated with the proportion of viable microspores present in the P2 polygon, in particular at D0. IFC quickly and precisely measures the stress efficiency on microspore populations. Two reactivity markers have been identified: cell viability and cell proportion in the $\mathrm{P} 2$ polygon, representing responsive microspores. IFC could therefore be considered as a reliable, robust and label-free marker of the stress efficiency for Pavon microspores and could be applied to other wheat genotypes or several plant species in androgenesis.

From D1 to D7, a second phase angle and an increase in amplitude were observed, corresponding to the second phase of embryogenic microspore development. These microspores actively divide and their cytoplasm densifies even more with the disappearance of vacuoles and vesicle formation [5]. The ribosome number considerably increases, just like the nuclei number, reflecting the high cellular activity of these microspores $[5,22,28,61]$. Thus, cytoplasm conductivity and permittivity of these microspores considerably change, as shown by the IFC analyses from D1 to D7. Heidmann et al. (2016) showed, with the use of beads of different sizes, that the amplitude increases observed in IFC represented an increase in cell size. This data is in agreement with our IFC results: the responsive microspores presented a significant size increase during the first days of culture, as confirmed by our microscopic observations and by several studies as well $[18,37]$. We can therefore hypothesize that these cytoplasmic rearrangements and this strong metabolic activity of embryogenic microspores are connected to the increase in the impedance phase angle and amplitude characteristics of the P3 polygon. More detailed studies could allow a finer characterization of the microspores contained in this polygon. Finally, we also highlighted a novel reactivity marker using the viable microspores detected in the P3 polygon by IFC technology to predict embryo yields in Pavon microspore suspensions. With an average of 13.5 embryos per 10,000 microspores, Pavon's embryo yields in our study fit with the other yields published for this genotype, which corroborate the reactivity threshold we set up $[62,63]$. To our knowledge, this is the first time 435 that embryo yields have been predicted early and reliably in androgenesis. The predictive mathematical model proposed for the Pavon genotype is highly significant and promising, and its robustness and precision will improve over time by adding more biological repetitions. Furthermore, additional studies are underway to adapt this model for embryo yield prediction to other wheat genotypes and other species in androgenesis. 
In conclusion, cellular and intracellular events that occur in responsive and embryogenic microspores throughout in vitro culture can be studied by using IFC. This technology provides a new approach to track microspore fates during androgenesis but is also an early, reliable and robust marker for efficiency evaluation of stress treatment. Using it, we demonstrated, for the first time, the possibility of early prediction of embryo yields of wheat microspore suspensions during the first seven days of in vitro culture.

\section{Acknowledgements}

The authors thank S. Letty for providing technical assistance, as well as M. Kloareg of the Kuzulia Company for her help and support in statistical analyses.

\section{Authors' contributions:}

Julie Canonge: Conceptualization, Methodology, Formal analysis, Investigation, Writing Original Draft, Writing - Review and Editing, Visualization. Murielle Philippot: 455 Conceptualization, Validation, Writing - Original Draft, Writing - Review and Editing. Catherine Leblanc: Conceptualization, Validation, Writing - Review and Editing. Philippe Potin: Conceptualization, Validation, Writing - Review and Editing, Supervision. Manuelle Bodin: Conceptualization, Validation, Writing - Original Draft, Writing - Review and Editing, Supervision, Project administration, Funding acquisition.

\section{Funding}

This work was supported by the European Regional Development Fund and by the Ministry of Higher Education, Research and Innovation in the context of the CIFRE $\mathrm{PhD}$ thesis n²017/0173 granted by ANRT, and by SECOBRA Recherches.

\section{Declaration of Interests}

The authors declare that they have no known financial interests or personal relationships that could have influenced the work reported in this paper.

\section{References}

[1] B. Echávarri, L. Cistué, Enhancement in androgenesis efficiency in barley (Hordeum vulgare L.) and bread wheat (Triticum aestivum $L$.) by the addition of dimethyl sulfoxide to the mannitol pretreatment medium, PCTOC. 125 (2016) 11-22. 
[2] S. Guha, S.C. Maheshwari, In vitro Production of Embryos from Anthers of Datura, Nature. 204 (1964) 497.

[3] M. Maluszynski, K.J. Kasha, B.P. Forster, I. Szarejko, eds., Doubled Haploid Production in Crop Plants, Springer Netherlands, Dordrecht, 2003.

[4] A. Touraev, O. Vicente, E. Herberle-Bors, Initiation of microspore embryogenesis by stress, Trends Plant Sci. 2 (1997) 297-302.

[5] A. Indrianto, I. Barinova, E. Herberle-Bors, Tracking individual wheat microspores in vitro: identification of embryogenic microspores and body axis formation in the embryo, Planta. 212 (2001) 163-174.

[6] J.B.M. Custers, J.H.G. Cordewener, Y. Nöllen, H.J.M. Dons, M.M. Van Lockeren Campagne, Temperature controls both gametophytic and sporophytic development in microspore cultures of Brassica napus, Plant Cell Rep. 13 (1994) 267-271.

[7] B. Leroux, N. Carmoy, D. Giraudet, P. Potin, F. Larher, M. Bodin, Inhibition of ethylene biosynthesis enhances embryogenesis of cultured microspores of Brassica napus, Plant Biotechnol. Rep. 3 (2009) 347-353.

[8] M.B.M. Bruins, M. Rakoczy-Trojanowska, C.H.A. Snijders, Isolated microspore culture in wheat (Triticum aestivum L.): the effect of co-culture of wheat or barley ovaries on embryogenesis, Cereal Res. Commun. 24 (1996) 401-408.

[9] T. Hu, K.J. Kasha, Improvement of isolated microspore culture of wheat (Triticum aestivum L.) through ovary co-culture, Plant Cell Rep. 16 (1997) 520-525.

[10] A. Touraev, M. Pfosser, E. Heberle-Bors, The microspore: A haploid multipurpose cell, in: J.A. Callow (Ed.), Advances in Botanical Research, Academic Press, 2001, pp. 53109.

[11] S.L. Dwivedi, A.B. Britt, L. Tripathi, S. Sharma, H.D. Upadhyaya, R. Ortiz, Haploids: Constraints and opportunities in plant breeding, Biotechnol. Adv. 33 (2015) 812-829.

[12] S.K. Datta, Androgenic haploids: Factors controlling development and its application in crop improvement, Curr. Sci. 89 (2005) 1870-1878.

[13] A. Jähne, H. Lörz, Cereal microspore culture, Plant Sci. 109 (1995) 1-12.

[14] M. Kumari, H.J. Clarke, I. Small, K.H.M. Siddique, Albinism in Plants: A Major Bottleneck in Wide Hybridization, Androgenesis and Doubled Haploid Culture, Crit.

505 Rev. Plant Sci. 28 (2009) 393-409.

[15] A. Olmedilla, Microspore Embryogenesis, in: E.C. Pua and M.R. Davey (Eds.), Plant Developmental Biology - Biotechnological Perspectives, Springer, New York, 2010, pp. 27-44.

[16] P.S. Testillano, Microspore embryogenesis: targeting the determinant factors of stressinduced cell reprogramming for crop improvement, J. Exp. Bot. 70 (2019) 2965-29789.

[17] A. Rivas-Sendra, P. Corral-Martínez, R. Porcel, C. Camacho-Fernández, A. CalabuigSerna, J.M. Seguí-Simarro, Embryogenic competence of microspores is associated with their ability to form a callosic, osmoprotective subintinal layer, J. Exp. Bot. 70 (2019) 1267-1281.

515 [18] K.P. Pauls, J. Chan, G. Woronuk, D. Schulze, J. Brazolot, When microspores decide to become embryos - Cellular and molecular changes, Can. J. Bot. 84 (2006) 668-678.

[19] R.A. Sánchez-Díaz, A.M. Castillo, M.P. Vallés, Microspore embryogenesis in wheat: new marker genes for early, middle and late stages of embryo development, Plant Reprod. 26 (2013) 287-296.

520 [20] A. Touraev, A. Indrianto, I. Wratschko, O. Vicente, E. Herberle-Bors, Efficient microspore embryogenesis in wheat (Triticum aestivum L.) induced by starvation at high temperatures., Sex. Plant Reprod. 9 (1996) 209-215. 
[21] S.F. Maraschin, M. Vennik, G.E.M. Lamers, H.P. Spaink, M. Wang, Time-lapse tracking of barley androgenesis reveals position-determined cell death within proembryos, Planta. 220 (2005) 531-540.

[22] J.M. Seguí-Simarro, F. Nuez, How microspores transform into haploid embryos: changes associated with embryogenesis induction and microspore-derived embryogenesis, Physiol. Plant. 134 (2008) 1-12.

[23] S.K. Raina, S.T. Irfan, High-frequency embryogenesis and plantlet regeneration from isolated microspores of indica rice, Plant Cell Rep. 17 (1998) 957-962.

[24] M.A.M. Zaki, H.G. Dickinson, Microspore-derived embryos in Brassica: the significance of division symmetry in pollen mitosis I to embryogenic development, Sex. Plant Reprod. 4 (1991) 48-55.

[25] A. Touraev, M. Pfosser, O. Vicente, E. Heberle-Bors, Stress as the major signal controlling the developmental fate of tobacco microspores: towards a unified model of induction of microspore/pollen embryogenesis, Planta. 200 (1996).

[26] D.E.S. Daghma, J. Kumlehn, G. Hensel, T. Rutten, M. Melze, Time-lapse imaging of the initiation of pollen embryogenesis in barley (Hordeum vulgare L.), J. Exp. Bot. 63 (2012) 6017-6021.

540 [27] M. Soriano, L. Hui, K. Boutilier, Microspore embryogenesis: establishment of embryo identity and pattern in culture, Plant Reprod. 26 (2013) 181-196.

[28] F.J. Bonet, A. Olmedilla, Structural changes during early embryogenesis in wheat pollen, Protoplasma. 211 (2000) 94-102.

[29] B. Barnabás, B. Obert, G. Kovács, Colchicine, an efficient genome-doubling agent for maize (Zea mays L.) microspores cultured in anthero, Plant Cell Rep. 18 (1999) 858862.

[30] J.M. Gonzalez, N. Jouve, Microspore development during in vitro androgenesis in triticale, Biol. Plant. 49 (2005) 23-28.

[31] X. Tang, Y. Liu, Y. He, L. Ma, M.-X. Sun, Exine dehiscing induces rape microspore polarity, which results in different daughter cell fate and fixes the apical-basal axis of the embryo, J. Exp. Bot. 64 (2013) 215-228.

[32] D.S. Hassawi, R.G. Sears, G.H. Liang, Microspore development in the anther culture of wheat (Triticum aestivum L.)., Cytologia (Tokyo). 55 (1990) 475-478.

[33] C. Gervais, W. Newcomb, D.H. Simmonds, Rearrangement of the actin filament and microtubule cytoskeleton during induction of microspore embryogenesis in Brassica napus L. cv. Topas, Protoplasma. 213 (2000) 194-202.

[34] D. Schulze, K.P. Pauls, Flow cytometric analysis of cellulose tracks development of embryogenic Brassica cells in microspore cultures, New Phytol. 154 (2002) 249-254.

[35] F.M. Ribalta, J.S. Croser, S.J. Ochatt, Flow cytometry enables identification of sporophytic eliciting stress treatments in gametic cells, J. Plant Physiol. 169 (2012) 104110.

[36] P. Corral-Martínez, A. Driouich, J.M. Seguí-Simarro, Dynamic Changes in Arabinogalactan-Protein, Pectin, Xyloglucan and Xylan Composition of the Cell Wall During Microspore Embryogenesis in Brassica napus, Front. Plant Sci. 10 (2019) 332.

[37] D.E.S. Daghma, G. Hensel, T. Rutten, M. Melze, J. Kumlehn, Cellular dynamics during early barley pollen embryogenesis revealed by time-lapse imaging, Fontiers Plant Sci. 5 (2014) 17-30.

[38] K.C. Cheung, M.D. Berardino, G. Schade-Kampmann, M. Hebeisen, A. Pierzchalski, J. Bocsi, A. Mittag, A. Tárnok, Microfluidic impedance-based flow cytometry, Cytometry A. 77A (2010) 648-666.

[39] S. Gawad, L. Schild, Ph. Renaud, Micromachined impedance spectroscopy flow cytometer for cell analysis and particle sizing, Lab. Chip. 1 (2001) 76-82. 
[40] F. David, M. Hebeisen, G. Schade, E. Franco-Lara, M.D. Berardino, Viability and membrane potential analysis of Bacillus megaterium cells by impedance flow cytometry, Biotechnol. Bioeng. 109 (2012) 483-492.

[41] C. Opitz, G. Schade, S. Kaufmann, M. Di Berardino, M. Ottiger, S. Grzesiek, Rapid determination of general cell status, cell viability, and optimal harvest time in eukaryotic cell cultures by impedance flow cytometry, Appl. Microbiol. Biotechnol. 103 (2019) 8619-8629.

[42] A. Pierzchalski, M. Hebeisen, A. Mittag, J. Bocsi, M.D. Berardino, A. Tarnok, Labelfree hybridoma cell culture quality control by a chip-based impedance flow cytometer, Lab. Chip. 12 (2012) 4533-4543.

[43] S. Crocetti, C. Beyer, G. Schade, M. Egli, J. Fröhlich, A. Franco-Obregón, Low Intensity and Frequency Pulsed Electromagnetic Fields Selectively Impair Breast Cancer Cell Viability, PLoS ONE. 8 (2013) e72944.

[44] A.R. Collins, B. Annangi, L. Rubio, R. Marcos, M. Dorn, C. Merker, I. Estrela-Lopis, M.R. Cimpan, M. Ibrahim, E. Cimpan, M. Ostermann, A. Sauter, N.E. Yamani, S. Shaposhnikov, S. Chevillard, V. Paget, R. Grall, J. Delic, F.G.- de-Cerio, B. SuarezMerino, V. Fessard, K.N. Hogeveen, L.M. Fjellsbø, E.R. Pran, T. Brzicova, J. Topinka, M.J. Silva, P.E. Leite, A.R. Ribeiro, J.M. Granjeiro, R. Grafström, A. Prina-Mello, M. Dusinska, High throughput toxicity screening and intracellular detection of nanomaterials, WIREs - Nanomedicine Nanobiotechnology. 9 (2017) e1413.

[45] I. Heidmann, G. Schade-Kampmann, J. Lambalk, M. Ottiger, M. Di Berardino, Impedance Flow Cytometry: A Novel Technique in Pollen Analysis, PLOS ONE. 11 (2016) e0165531.

[46] J. Xu, N. Driedonks, M.J.M. Rutten, W.H. Vriezen, G.-J. de Boer, I. Rieu, Mapping quantitative trait loci for heat tolerance of reproductive traits in tomato (Solanum lycopersicum), Mol. Breed. 37 (2017) 37-58.

[47] I. Heidmann, M. Di Berardino, Impedance Flow Cytometry as a Tool to Analyze Microspore and Pollen Quality, in: A. Schmidt (Ed.), Plant Germline Dev. Methods Protoc., Springer New York, New York, 2017, pp. 339-354.

[48] P. Vergne, I. Delvallee, C. Dumas, Rapid Assessment of Microspore and Pollen Development Stage in Wheat and Maize Using Dapi and Membrane Permeabilization, Stain Technol. 62 (1987) 299-304.

[49] J. De Buyser, P. Touraine, F. J'aïti, R. Haïcour, E. Picard, Haplodiploïdisation par culture de microspores isolées de blé in vitro, in: Biotechnol. Végétales Tech. Lab., Tec \& Doc, Lavoisier, Paris, 2002, pp. 257-273.

[50] M.Y. Zheng, W. Liu, Y. Weng, E. Polle, C.F. Konzak, Production of doubled haploids in wheat (Triticum aestivum L.) through microspore embryogenesis triggered by inducer chemicals, in: Doubled Haploid Prod. Crop Plants, Kluwer Academic Publishers, pp. 83-94.

[51] J. Heslop-Harrison, Y. Heslop-Harrison, Evaluation of pollen viability by enzymatically induced fluorescence; intracellular hydrolysis of fluorescein diacetate, Stain Technol. 45 (1970) 115-120.

[52] J. Josse, F. Husson, missMDA: A Package for Handling Missing Values in Multivariate Data Analysis, J. Stat. Softw. 70 (2016) 1-31.

[53] T.L. Reynolds, A Cytological Analysis of Microspores of Triticum aestivum (Poaceae) During Normal Ontogeny and Induced Embryogenic Development, Am. J. Bot. 80 (1993) 569-576.

620 [54] M. Rodriguez-Serrano, I. Barany, D. Prem, M.-J. Coronado, M.C. Risueno, P.S. Testillano, NO, ROS, and cell death associated with caspase-like activity increase in stress-induced microspore embryogenesis of barley, J. Exp. Bot. 63 (2012) 2007-2024. 
[55] Y. Pérez-Pérez, I. Bárány, E. Berenguer, E. Carneros, M.C. Risueño, P.S. Testillano, Modulation of autophagy and protease activities by small bioactive compounds to reduce cell death and improve stress-induced microspore embryogenesis initiation in rapeseed and barley, Plant Signal. Behav. 14 (2019) 1559577.

[56] J.M. Seguí-Simarro, P.S. Testillano, M.C. Risueño, Hsp70 and Hsp90 change their expression and subcellular localization after microspore embryogenesis induction in Brassica napus L., J. Struct. Biol. 142 (2003) 379-391.

630 [57] I. Bárány, E. Berenguer, M.-T. Solís, Y. Pérez-Pérez, M.E. Santamaría, J.L. Crespo, M.C. Risueño, I. Díaz, P.S. Testillano, Autophagy is activated and involved in cell death with participation of cathepsins during stress-induced microspore embryogenesis in barley, J. Exp. Bot. 69 (2018) 1387-1402.

[58] P.S. Testillano, Stress-Induced Microspore Embryogenesis in Crop Plants: Cell Totipotency Acquisition and Embryo Development, in: Prog. Bot., Springer, Cham, 2018, pp. 1-15.

[59] M.R. Malik, F. Wang, J.M. Dirpaul, N. Zhou, P.L. Polowick, A.M.R. Ferrie, J.E. Krochko, Transcript Profiling and Identification of Molecular Markers for Early Microspore Embryogenesis in Brassica napus, Plant Physiol. 144 (2007) 134-154.

[60] P. Corral-Martínez, V. Parra-Vega, J.M. Seguí-Simarro, Novel features of Brassica napus embryogenic microspores revealed by high pressure freezing and freeze substitution: evidence for massive autophagy and excretion-based cytoplasmic cleaning, J. Exp. Bot. 64 (2013) 3061-3075.

[61] P.S. Testillano, P. González-Melendi, M.J. Coronado, J.M. Seguí-Simarro, M.A. Moreno-Risueño, M.C. Risueño, Differentiating plant cells switched to proliferation remodel the functional organization of nuclear domains, Cytogenet. Genome Res. 109 (2005) 166-174.

[62] Z. Labbani, N. Richard, J. De Buyser, E. Picard, Plantes chlorophylliennes de blé dur obtenues par culture de microspores isolées : importance des prétraitements, C. R. Biol. 328 (2005) 713-723.

[63] L.B. Poersch-Bortolon, S.M.M. Scagliusi, E. Yamazaki-Lau, M.H. Bodanese-Zanettini, Androgenic response of Brazilian wheat genotypes to different pretreatments of spikes and to a gelling agent, Pesqui. Agropecuária Bras. 51 (2016) 1839-1847. 

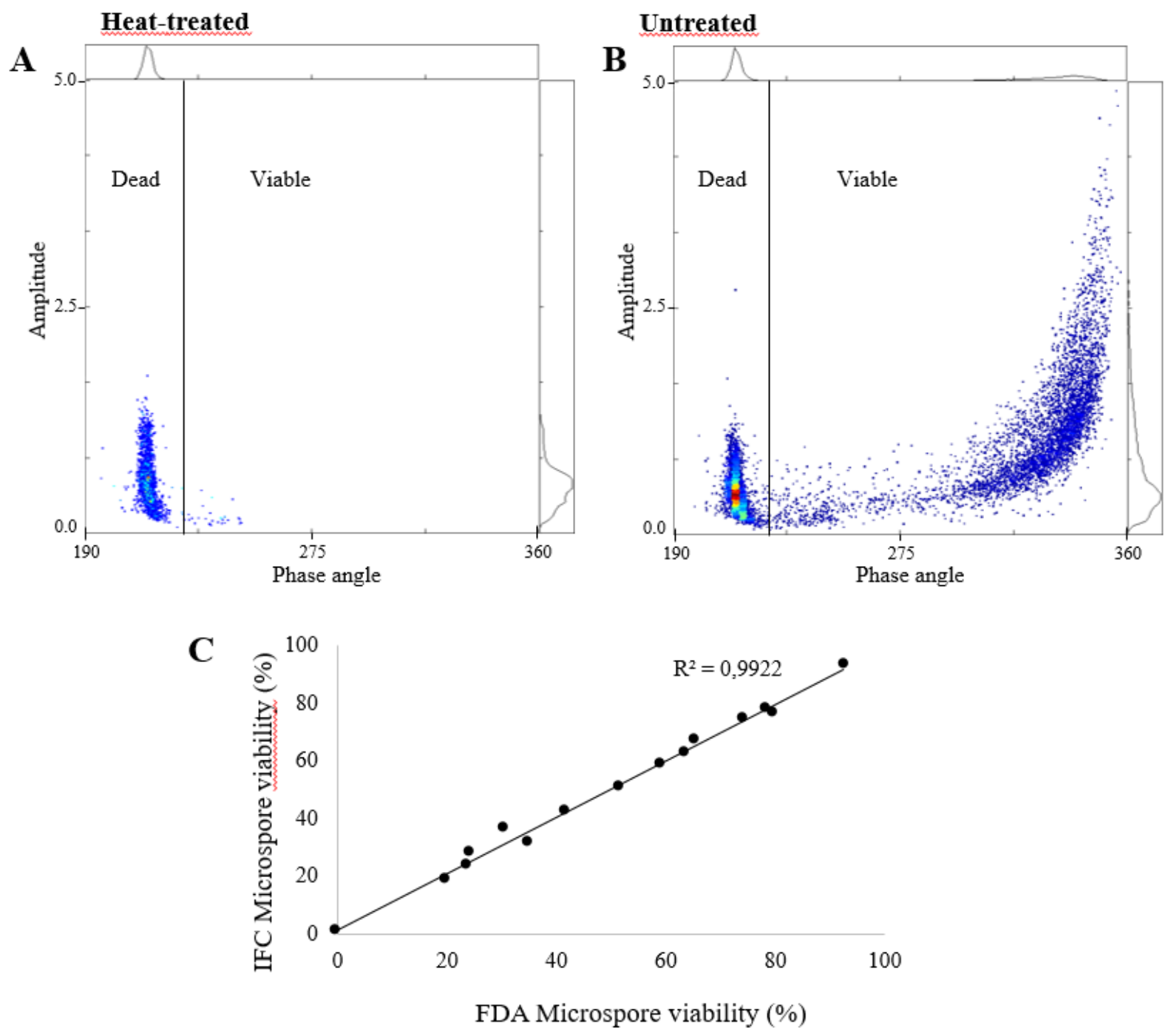

Fig. 1. IFC discrimination of viable and dead cells in microspore suspensions.

Amphasoft dot plots represent heat-treated (A) and untreated (B) microspore suspensions at D1 analyzed at $12 \mathrm{MHz}$. Vertical gating differentiates dead microspores (left) with a low phase angle, and viable microspores (right) with a high phase angle. (C) Correlation between IFC data at $12 \mathrm{MHz}$ and viability measurement with FDA staining of 15 mixed samples of fresh and heat-treated microspores. ( $\mathrm{p}$-value $\left.<0.05 ; \mathrm{r}^{2}=0.9922\right)$. Each point represents one sample analyzed by IFC and microscopy; no technical replicate was done. 
Gametophytic pathway

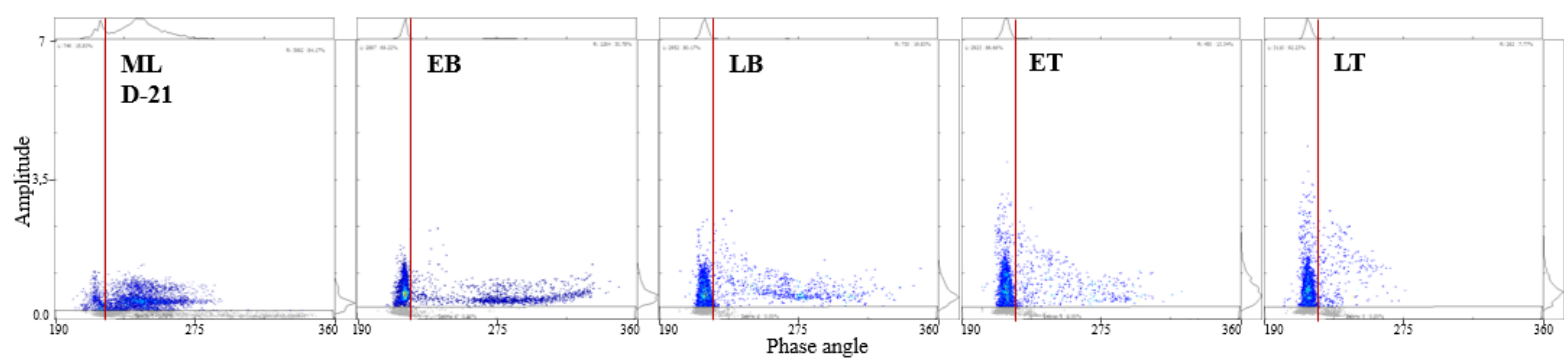

Sporophytic pathway

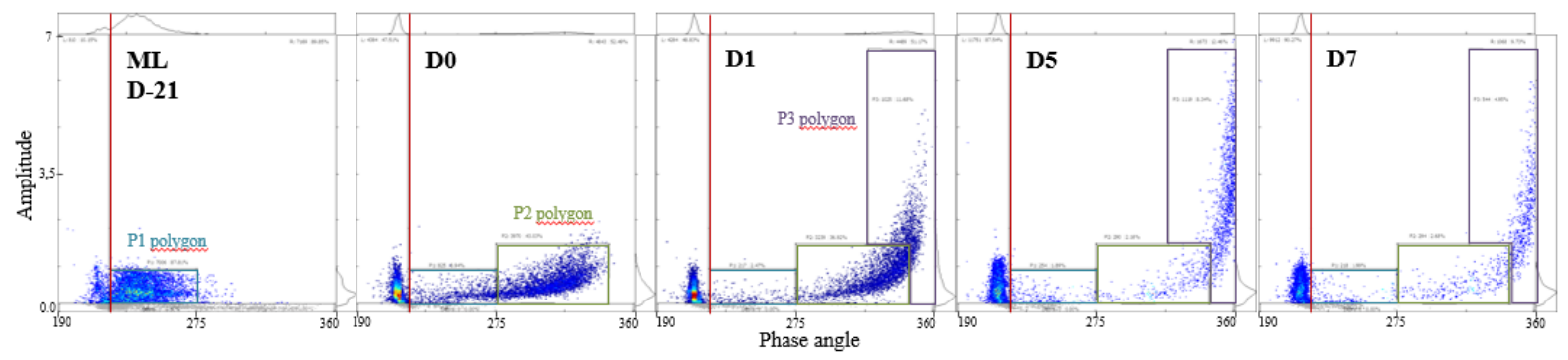

Fig. 2. Major stages of gametophytic and sporophytic developmental pathways of Pavon microspores analyzed by IFC.

680 Gametophytic pathway: ML: mid-to-late uninucleate; EB: early binucleate; LB: late uninucleate; ET: early trinucleate; LT: late trinucleate. Sporophytic pathway: microspore cultures at D-21, D0, D1, D5 and D7 are shown. Microspores at ML/D-21 represent the common stage between gametophytic and sporophytic development. For each graph, the vertical red line differentiates dead (left) and viable (right) microspores in the population. P1, $\mathrm{P} 2$ and $\mathrm{P} 3$ polygons are represented in blue, green and purple, respectively. 

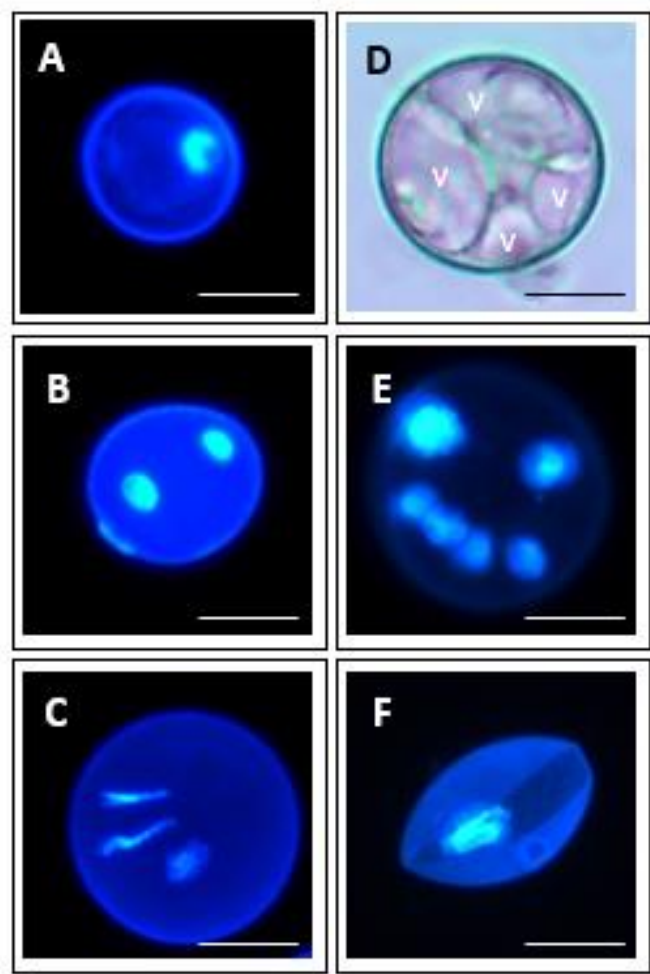

A

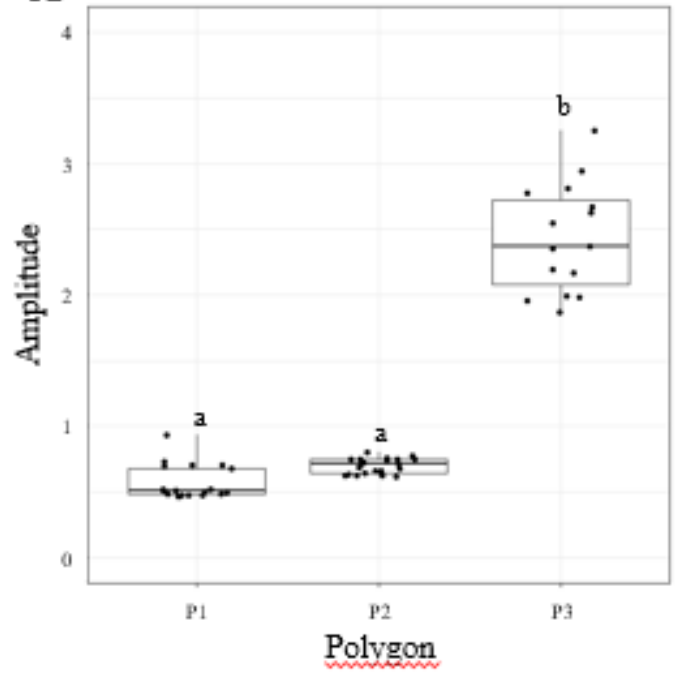

Fig. 3. Developmental stages of gametophytic and sporophytic pathways of Pavon microspores observed in microscopy.

(A) Uninucleate microspore; (B) Binucleate microspore; (C) Pollen grain; (D) Star-like microspore ( $\mathrm{v}=$ vacuole); (E) Multinucleate microspore; (F) Plasmolyzed microspore. Microspores were observed with fluorescent DAPI staining of nuclei or using visible light microscopy. Bars $=20 \mu \mathrm{m}$.

Fig. 4. Amplitude and phase angle medians of the three polygons delimiting dot plots in IFC. Amplitude (A) and phase angle (B) medians of P1, P2 and P3 polygons were obtained from at least five microspore suspensions analyzed by IFC at $12 \mathrm{MHZ}$ at D-21, D0 and D5, respectively. Each point represents the median of the microspore population inside the polygon for one measurement. Within a graph, boxes with the same letter are not significantly different (Tukey's test, $\mathrm{p}<0.05)$. 


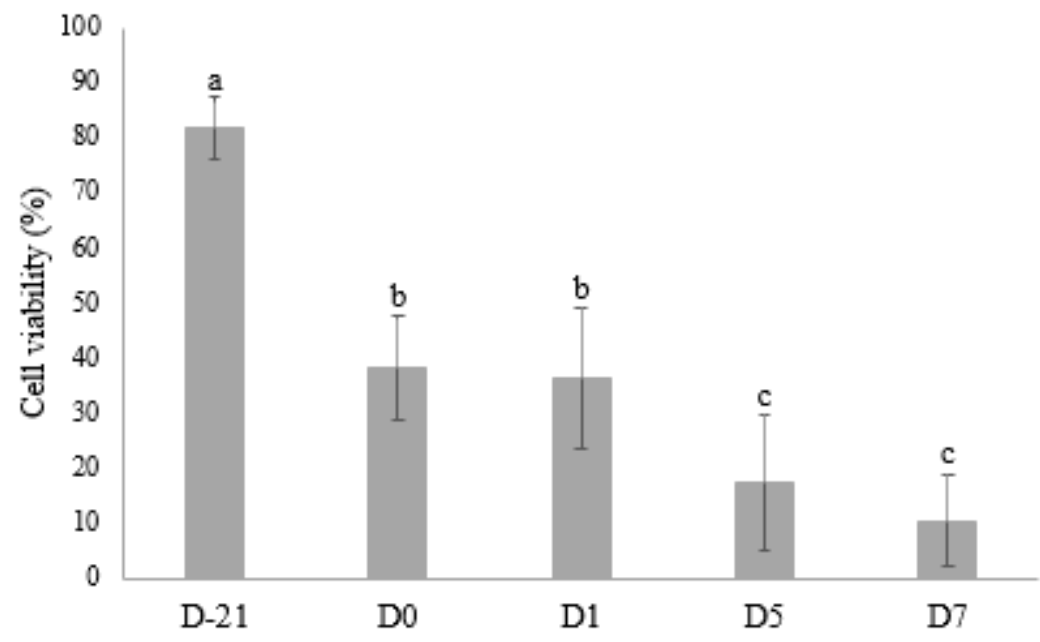

Fig. 5. Evolution of microspore viability during the androgenesis process.

Mean percentages of microspore viability from D-21 to D7 were analyzed by IFC at $12 \mathrm{MHZ}$ from at least five biological replicates. Each point represents an average of technical triplicates. Standard deviations (shown by error bars) were determined for each day of analysis. Data with the same letter are not significantly different (Tukey's test, $\mathrm{p}<0.05$ ).

A

\begin{tabular}{c|cccccc}
\multicolumn{1}{c}{} & & D-2l & D0 & D1 & D5 & D7 \\
\hline \multirow{4}{*}{ DAPI } & Uninucleate & $97.6^{\mathrm{b}}$ & $78.7^{\mathrm{a}}$ & $78.1^{\mathrm{a}}$ & $76.5^{\mathrm{a}}$ & $78.5^{\mathrm{a}}$ \\
& Binucleate & $2.4^{\mathrm{a}}$ & $21.1^{\mathrm{b}}$ & $20.2^{\mathrm{b}}$ & $20.8^{\mathrm{b}}$ & $19.5^{\mathrm{b}}$ \\
& Trinucleate & $0^{\mathrm{a}}$ & $0.2^{\mathrm{ab}}$ & $1.1^{\mathrm{ab}}$ & $1.3^{\mathrm{b}}$ & $1.0^{\mathrm{ab}}$ \\
& Multinucleate & $0^{\mathrm{a}}$ & $0^{\mathrm{a}}$ & $0.6^{\mathrm{ab}}$ & $1.4^{\mathrm{b}}$ & $1.0^{\mathrm{ab}}$ \\
\hline Visible & Star-like microspore & - & 41.5 & - & - & - \\
\hline \multirow{5}{*}{ IFC } & Non-viable microspore & $18.4^{\mathrm{a}}$ & $60.2^{\mathrm{b}}$ & $62.5^{\mathrm{b}}$ & $82.6^{\mathrm{c}}$ & $89.6^{\mathrm{c}}$ \\
& Viable microspore in P1 & $80.1^{\mathrm{b}}$ & $5.7^{\mathrm{a}}$ & $2.2^{\mathrm{a}}$ & $2.4^{\mathrm{a}}$ & $2.0^{\mathrm{a}}$ \\
& Viable microspore in P2 & $1.1^{\mathrm{a}}$ & $33.8^{\mathrm{b}}$ & $25.2^{\mathrm{b}}$ & $8.4^{\mathrm{a}}$ & $5.2^{\mathrm{a}}$ \\
& Viable microspore in P3 & $0^{\mathrm{a}}$ & $0.2^{\mathrm{a}}$ & $10.3^{\mathrm{c}}$ & $6.4^{\mathrm{bc}}$ & $3.1^{\mathrm{ab}}$
\end{tabular}

B

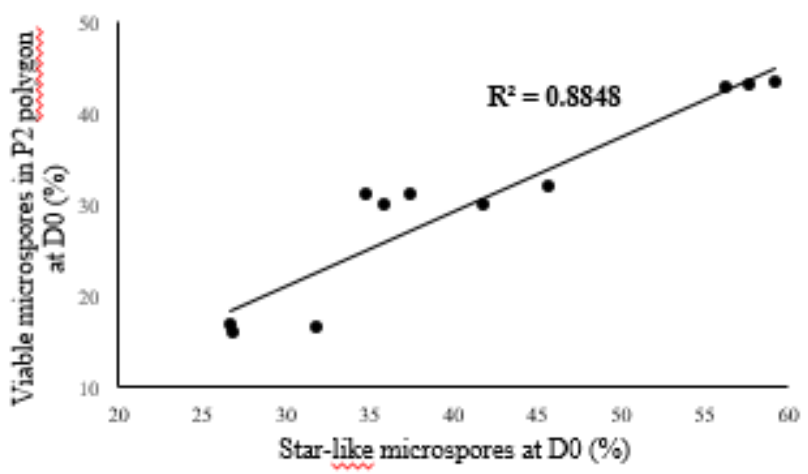

725 Fig. 6. Comparison of microspore population descriptions throughout the androgenesis process obtained by microscopy and IFC analyses. (A) Percentages of cell types observed in microspore 
suspensions at different culture times, established by counting with DAPI staining and visible microscopy, and IFC analyses at $12 \mathrm{MHz}$. Percentages were calculated from a minimum of five biological replicates. For IFC data, technical triplicates were performed and averaged for each day of analysis. For each category, data with the same letter are not significantly different (Tukey's test, $\mathrm{p}<0.05$ ). (B) Correlation between star-like microspore proportions and the percentage of viable microspores in the $\mathrm{P} 2$ polygon at D0 using a linear regression analysis ( $\mathrm{p}$ value $\left.<0.05 ; \mathrm{y}=0.82 \mathrm{x}-3.5 ; \mathrm{r}^{2}=0.8848\right)$. Four biological replicates were analyzed by both methods; each point represented a technical replicate.

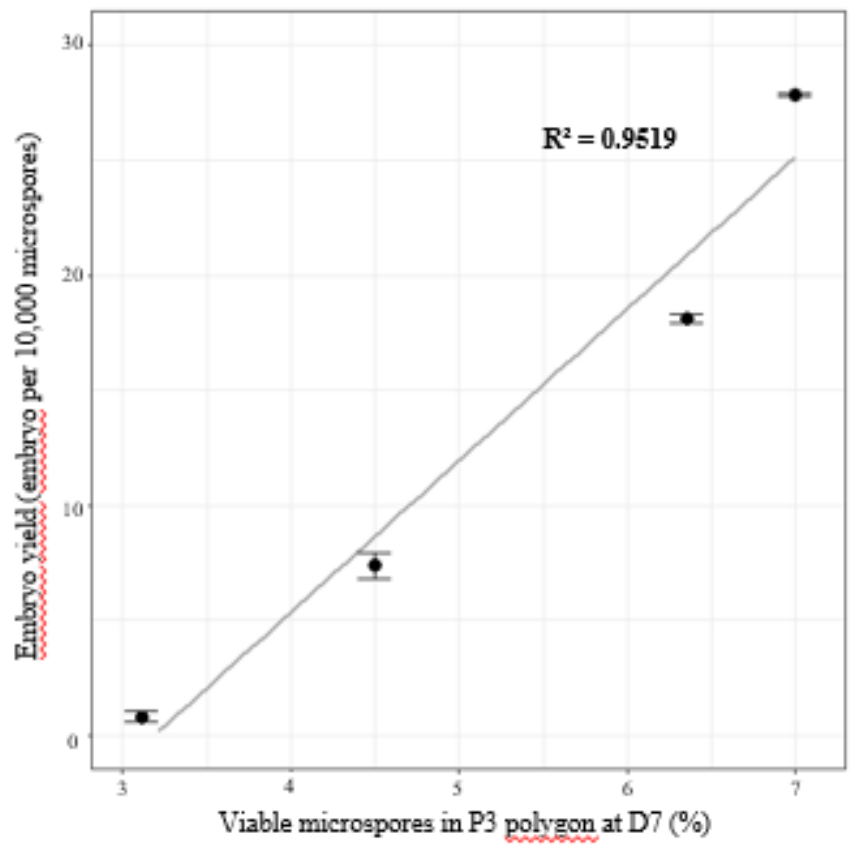

Fig. 7. Early prediction of embryo yield for the Pavon genotype with IFC measurement.

Application of a prediction model for the linear correlation between the percentage of viable microspores in the P3 polygon at D7 and the embryo yield. Four biological replicates were used to establish this mathematical model, with the average of technical triplicates for IFC measurements represented by error bars. The coefficients of this prediction model were obtained by adjusting the linear model using R software (linear equation: $y=6.539 x-20.827$; p-value $<0.05)$. 